\title{
BMJ Open Management strategies for chronic rhinosinusitis: a qualitative study of GP and ENT specialist views of current practice in the UK
}

\author{
Jane Vennik, ${ }^{1}$ Caroline Eyles, ${ }^{1}$ Mike Thomas, ${ }^{1}$ Claire Hopkins, ${ }^{2}$ Paul Little, ${ }^{1}$ \\ Helen Blackshaw, ${ }^{3}$ Anne Schilder, ${ }^{3}$ Jim Boardman, ${ }^{4}$ Carl M Philpott ${ }^{5,6}$
}

To cite: Vennik J, Eyles C, Thomas M, et al. Management strategies for chronic rhinosinusitis: a qualitative study of GP and ENT specialist views of current practice in the UK. BMJ Open 2018;8:e022643. doi:10.1136/ bmjopen-2018-022643

- Prepublication history and additional material for this paper are available online. To view these files, please visit the journal online (http://dx.doi. org/10.1136/bmjopen-2018022643).

Received 28 February 2018 Revised 24 October 2018 Accepted 25 0ctober 2018

Check for updates

(C) Author(s) (or their employer(s)) 2018. Re-use permitted under CC BY-NC. No commercial re-use. See rights and permissions. Published by BMJ.

For numbered affiliations see end of article.

Correspondence to

Dr Jane Vennik;

j.vennik@soton.ac.uk

\section{ABSTRACT}

Objectives To explore general practitioner (GP) and ears, nose and throat (ENT) specialist perspectives of current treatment strategies for chronic rhinosinusitis (CRS) and care pathways through primary and secondary care.

Design Semi-structured qualitative telephone interviews as part of the MACRO programme (Defining best Management for Adults with Chronic Rhinosinusitis) Setting Primary care and secondary care ENT outpatient clinics in the UK.

Participants Twelve GPs and 9 ENT specialists consented to in-depth telephone interviews. Transcribed recordings were managed using NVivo software and analysed using inductive thematic analysis.

Main outcome measures Healthcare professional views of management options and care pathways for CRS.

Results GPs describe themselves as confident in recognising CRS, with the exception of assessing nasal polyps. In contrast, specialists report common missed diagnoses (eg, allergy; chronic headache) when patients are referred to ENT clinics, and attribute this to the limited ENT training of GPs. Steroid nasal sprays provide the foundation of treatment in primary care, although local prescribing restrictions can affect treatment choice and poor adherence is perceived to be the causes of inadequate symptom control. Symptom severity, poor response to medical treatment and patient pressure drive referral, although there is uncertainty about optimal timing. Treatment decisions in secondary care are based on disease severity, polyp status, prior medical treatment and patient choice, but there is major uncertainty about the place of longer courses of antibiotics and the use of oral steroids. Surgery is regarded as an important treatment option for patients with severe symptoms or with nasal polyps, although timing of surgery remains unclear, and the uncertainty about net long-term benefits of surgery makes balancing of benefits and risks more difficult.

Conclusions Clinicians are uncertain about best management of patients with CRS in both primary and secondary care and practice is varied. An integrated care pathway for CRS is needed to improve patient management and timely referral.
Strengths and limitations of this study

- The interviews permitted exploration of diagnosis, treatment and management strategies for patients with chronic rhinosinusitis (CRS) through primary and secondary care.

- Participants were sampled from different geographical areas of England and Scotland, enhancing transferability of the study findings.

- The inclusion of generalist and specialist views provided a better understanding of CRS management from multiple perspectives.

- Including other clinicians such as ears, nose and throat specialty doctors and primary care nurse practitioners may have provided an additional viewpoint.

- Patient views and experiences are not included but are presented elsewhere.

\section{INTRODUCTION}

Chronic rhinosinusitis (CRS) is a prevalent chronic inflammatory condition of the nose and paranasal sinuses which significantly affects the health and quality of life of patients, ${ }^{2}$ and contributes a significant burden to the National Health Service healthcare resources. CRS is defined in the European Position Paper on Rhinosinusitis (EPOS 2012) ${ }^{3}$ by the presence of at least two symptoms, one of which must be either nasal congestion or nasal discharge together with facial pain/pressure and/or anosmia lasting for $>12$ weeks. This is then further qualified by the presence (CRSwNP) or absence of nasal polyps (CRSsNP). Based on the symptom and duration definition alone, prevalence rates are estimated at $10 \%$ in the UK. ${ }^{4}$ Longitudinal data from the Clinical Practice Research Datalink suggests that $1 \%$ of British adults receive treatment each year in primary care, resulting in multiple GP consultations and medical prescriptions. ${ }^{5}$ There is significant 
onward referral to specialist ears, nose and throat (ENT) services leading to 120000 outpatient appointments and 40000 sinus operations annually in England and Wales. ${ }^{6}$

To date, there are no National Institute for Health and Care Excellence (NICE) guidelines for managing patients with CRS. International ${ }^{7}$ and European (EPOS) guidelines $^{3}$ are present, however, awareness and uptake in UK practice is unclear. Commissioning guidelines ${ }^{8}$ for management of rhinosinusitis were developed in collaboration with the Royal College Of Surgeons England and NICE, but local compliance with guidelines is variable and access to specialist care is restricted in some areas. Intranasal corticosteroids (INCS) and nasal irrigation are strongly recommended based on high-quality evidence, ${ }^{9-11}$ however there are uncertainties about uptake and compliance in primary care. Most consultations with general practitioners (GPs) for acute or chronic rhinosinusitis result in an antibiotic prescription despite insufficient evidence for routine use. ${ }^{12}$ Uncertainties in secondary care result in a fivefold variation in surgical rates around the $\mathrm{UK}^{6}$ and there is conflicting evidence for the use of long-term antibiotics. ${ }^{13}$

The views and experiences of healthcare professionals involved in the CRS patient pathway have yet to be investigated in depth. As yet, no studies have explored GP practice variation in terms of knowledge of the guidelines, treatment decisions and referral criteria, and there is limited understanding of ENT specialist views and experiences of treating patients with CRS.

The MACRO programme (Defining best Management for Adults with Chronic Rhinosinusitis) ${ }^{14}$ is the National Institute for Health Research (NIHR) funded programme of research designed to establish best practice for CRS management in adults across primary and secondary care. This paper reports a qualitative interview study, conducted as part of the MACRO programme, exploring GP and ENT specialist views of current treatment strategies and care pathways for patients with CRS through primary and secondary care.

\section{METHODS}

\section{Design}

A qualitative interview study was conducted with a purposeful sample of GPs and ENT specialists between January and April 2017.

\section{Participants and procedures}

GPs were approached through the NIHR Clinical Research Network in regions of Wessex, Eastern and South East England. Fifteen interested GPs with experience of treating patients with CRS responded to the study team and were purposefully sampled for a range of characteristics including gender, GP experience, practice location and practice population demographics.

ENT specialists with experience of treating patients with CRS were recruited through an email to the membership of ENT-UK (the professional body representing ENT surgeons). Thirty-one ENT specialists from England and Scotland expressed an interest in participating and were purposefully sampled for a range of demographics (location, time in practice) and ENT subspecialty interest (including generalists and rhinologists).

\section{Interviews}

A trained interviewer (JV) conducted semi-structured telephone interviews with participants each lasting approximately $25-55 \mathrm{~min}$. JV (female) is a postdoctoral research fellow for the MACRO programme, trained in qualitative research methods and with previous experience of ENT and primary care research, who was not previously known to the interview participants. Telephone interviews were employed to allow inclusion of participants from a wide geographical area. Each participant gave verbal consent prior to commencing the interview. An interview guide (see online supplementary appendix 1) was developed through collaboration and input from the wider MACRO research team, and subsequently piloted with an ENT specialist and minor modifications made to reflect any issues that arose. The guide was used to direct but not constrain the interviews and was sufficiently flexible to allow exploration of unexpected topics and themes. Field notes were used to facilitate interpretation and contextualisation of the interviews.

GPs and ENT specialists were asked open-ended questions about their views of the diagnosis and management of CRS, knowledge and implementation of CRS guidelines, perceptions of the evidence base and experiences of practical decision-making in the management of patients with CRS. The interviews were audio-recorded and transcribed verbatim in preparation for analysis. Our epistemological position is one of subtle realism, and a pragmatic approach was adopted in this study.

\section{Analysis}

Inductive thematic analysis ${ }^{15}$ was used to analyse the interview transcripts, using NVIVO 11 to facilitate data management. Each participant group (GPs and ENT surgeons) were analysed independently. Each analysis commenced with familiarisation and immersion in the data itself, leading to identification of initial patterns and themes within the data set. Descriptive codes were then used to label sections of the data. A number of transcripts were coded by more than one researcher (JV and CE). which brings multiple perspectives to the analysis and provides an opportunity to discuss coding decisions at an early stage. Codes were then developed and refined as analysis progressed, and where uncertainties arose, team discussions facilitated agreement and adjustment. These codes were then linked together, grouped, refined and re-labelled resulting in a set of themes and subthemes for each participant group. Following an iterative process of comparing and contrasting the two datasets, a set of overarching themes and subthemes were developed which systematically and thoroughly explained the data. Data collection and analysis took place concurrently, and interviews continued until data saturation was achieved. 


\begin{tabular}{|c|c|}
\hline GP characteristics & $\mathrm{n}=12$ \\
\hline Years in general practice (median, range) & $20(2-29)$ \\
\hline Male & 8 \\
\hline Practice list size mean (range) & $9967(4758-18$ 571) \\
\hline $\begin{array}{l}\text { Practice deprivation decile (where } 1 \text { is most deprived, } \\
10 \text { is least deprived) median (range) }\end{array}$ & $9(3-10)$ \\
\hline \multicolumn{2}{|l|}{ Practice location } \\
\hline Rural town and fringe & 4 \\
\hline Rural village and dispersed & 1 \\
\hline Urban city and town & 4 \\
\hline Urban major conurbation & 3 \\
\hline ENT specialist characteristics & $n=9$ \\
\hline $\begin{array}{l}\text { Time (years) on specialist register (otolaryngology) } \\
\text { (median, range) }\end{array}$ & $19(8-21)$ \\
\hline Male & 8 \\
\hline \multicolumn{2}{|l|}{ Subspecialisation } \\
\hline General ENT surgeon & 3 \\
\hline Special interest in rhinology & 4 \\
\hline Consultant rhinologist & 2 \\
\hline \multicolumn{2}{|l|}{ Location } \\
\hline Yorkshire and Humberside & 1 \\
\hline Midlands & 1 \\
\hline London & 1 \\
\hline South East & 4 \\
\hline Scotland & 2 \\
\hline
\end{tabular}

ENT, ears, nose and throat.

\section{Patient involvement}

A patient contributor (JB) from the MACRO programme management group provided input into the design of the study from the patient perspective, and contributed to the development of the interview topic guide.

\section{RESULTS}

\section{Participants}

A total of 12 GPs participated in the study. Eight (76\%) were male with median duration in general practice of 20 years (range 2-29). Included GPs were from rural and urban practices, from areas of differing social deprivation. Nine ENT specialists including general ENT surgeons and rhinologists also took part in a research interview; eight were male with a median time on the specialist register of 19 years (range 8-21). Full details are presented in table 1 .

\section{Themes}

Thematic analysis identified four main themes relating to the management of patients with CRS (table 2). Quotations are presented to illustrate the themes and subthemes, with details of the participants presented in parentheses.

\begin{tabular}{|c|c|}
\hline Theme & Subtheme \\
\hline 1. Diagnostic uncertainties & $\begin{array}{l}\text { Reaching a diagnosis } \\
\text { Distinguishing between subgroups }\end{array}$ \\
\hline 2. Selecting best management & $\begin{array}{l}\text { Treatment decisions } \\
\text { Initiation treatment in primary care } \\
\text { Further medical treatment options }\end{array}$ \\
\hline 3. Decision-making for surgery & $\begin{array}{l}\text { Implementing guidelines } \\
\text { Value of sinus surgery } \\
\text { Joint decision-making }\end{array}$ \\
\hline 4. Transition of care & $\begin{array}{l}\text { Factors affecting referral } \\
\text { Quality of referral }\end{array}$ \\
\hline
\end{tabular}

\section{Theme 1: diagnostic uncertainties}

Reaching a diagnosis

GPs describe themselves as confident in recognising CRS, making a diagnosis based on patient-reported history of symptoms and impact on quality of life, together with a basic physical examination of the nose. Duration of symptoms was described as an important diagnostic factor, although there is some uncertainty in primary care about the definition of chronicity.

Then I guess really the diagnosis comes down to the time course more than anything. Assuming that that cluster of symptoms is present, it comes down to the time course. (GP 04)

On the contrary, ENT specialists describe primary care diagnosis of CRS as of variable quality and precision, with many patients presenting at ENT clinics with a misdiagnosis.

So I think, therefore, their diagnostic ability for ENT conditions, broadly, and rhinosinusitis in particular, can be very poor. (ENT 01)

Uncertainties can arise when symptoms overlap with other conditions, such as chronic headaches and allergic rhinitis, which can lead to patients receiving ineffective treatments.

We see lots of patients who are referred with 'recurrent sinus infections' where their main presenting symptom may be facial pain or headache, and most of those patients, in my experience, with those symptoms, don't have rhinosinusitis. (ENT 02)

ENT specialists also describe a diagnostic challenge where there is a dissociation between patient symptom history and clinical findings.

There's a well-defined group of patients who have symptoms which are on history virtually indistinguishable from CRS who actually have nothing on nasal endoscopy, in other words completely normal nasal endoscopy, and when the CT scan comes back that's completely normal as well. (ENT 05) 


\section{Distinguishing between subgroups}

GPs report some difficulties in diagnosing patients with nasal polyps unless polyps are visible in the nasal cavity, due to the lack of diagnostic equipment in primary care.

Yes, with polyps, on the outset, if we have a patient with these symptoms, to sort of diagnose polyps is very difficult for us unless they've got a previous diagnosis. (GP 08)

In contrast, ENT specialists report the diagnosis of CRS without nasal polyps to be more challenging particularly when there is an absence of clinical findings on examination.

The diagnosis of chronic rhinosinusitis without nasal polyps is really, really difficult. As I say, some of them will actually have pus dripping from their sinus into the nose, and that's very easy, but the majority of them don't have that at all. (ENT 08)

\section{Theme 2: selecting best management}

Treatment decisions

GPs report using local guidelines, journal articles, online learning modules and GP educational sessions to keep up to date with current practices. However, GP update courses are described as often too general and cover a broad range of topics. Events provided by the local ENT department were described as extremely useful but were infrequent.

I tend to do a lot of online stuff, so I'll look at BMJ or RCGP learning. Sometimes, we get local events. We've not had a local ENT event recently, but we do get local consultants or specialist nurses, sometimes, come out and give us updates and guidelines. (GP 05)

GPs also describe ENT clinic letters as useful and informative about current practice for managing patients with CRS.

You get a flavour of reading letters and so you get an idea of what they recommend in their letters. (GP 09)

Nonetheless, ENT specialists expressed opinion that GPs have limited ENT training and some are poorly trained to recognise and understand symptoms and pathology of CRS.

Most GPs have never done any ENT jobs, ever, in their training. Most medical students don't get any ENT teaching, or very minimal ENT teaching, so you then have a sub-set of GPs who don't know any ENT unless it's what they've been told by their GP colleagues on the odd course they go to. (ENT 01)

\section{Initiating treatment in primary care}

INCS were commonly described as first-line treatment for patients with CRS, with the choice often associated with GP preference and experience. However, some GPs describe local prescribing restrictions where they are directed to prescribe lower cost nasal sprays such as beclomethasone, instead of more costly fluticasone, in the first instance.

There will pop up a box (on our computers) saying, 'This costs this much, why don't you use this one which costs only this much?' So we're constantly reminded to use cost effective things. (GP 02)

However, both GPs and ENT specialists suggested that patients did not always apply their nasal sprays correctly or did not comply with the treatment regimen over a longer period of time.

It's quite obvious that they have been sniffing too much and it's gone all the way to the back of the throat, and they're using it incorrectly, maybe because no one informed them of how to use it in the past. (GP 06)

GPs describe prescribing short courses of antibiotics for patients with acute infection, purulent discharge, febrile patients and for those who are most severely affected. However, some GP report feeling pressured by patients to prescribe antibiotics, especially if symptoms are particularly severe or the patient has responded to antibiotics in a previous episode.

The biggest challenge is patients wanting antibiotics because they think that will cure everything! (GP 05)

Most GPs reported limited use of saline irrigation in primary care. Despite recommendations from ENT, GPs were unfamiliar with the evidence and therefore unlikely to recommend them to patients, or patients were concerned about the cost of proprietary preparations.

ENT doctors recommend it (saline irrigation) to patients, but that's not something I'd recommend myself...I just wasn't aware if it was evidence-based, or if it was something we should be recommending. (GP 09)

\section{Further medical treatment options}

Some ENT specialists report the use of long-term antibiotics for patients with CRS, although there is uncertainty about which patients might benefit and the optimum length of treatment.

I think the issue is about what is the role of long-term antibiotics in rhinosinusitis and particularly the clarithromycin group of antibiotics? It is something I am aware of being used increasingly and I do now use in some patients. (ENT 02)

There is a mixed opinion about whether long-term antibiotics are appropriate for patients with CRS with nasal polyps, with some ENT specialists describing them as ineffective, and a lack of evidence for their routine use. Others, however, recount prescribing low-dose antibiotics 
alongside oral steroids for nasal polyps prior to consideration for surgery.

I don't use clarithromycin for polyps. I know some people do but I don't because from my reading of the literature and all that I don't really see any of the benefit. (ENT 07)

Most GPs reported being unfamiliar with long term antibiotic use for CRS in primary care, and some were concerned about the potential for side effects.

I don't have any experience of it. I'm not keen on long term courses of antibiotics, for this particular condition. (GP 07)

GPs described infrequent use of oral steroids in primary care. ENT specialists report the use of oral steroids in the preoperative pathway for patients with polyp disease. A rescue course of oral steroids is sometimes used for severe patients when symptoms are uncontrolled. Oral steroids were reported as rarely recommended for patients without nasal polyps.

I think most people would say that the polyp group need a lot more oral steroid and a lot less antibiotic, and the non-polyp group need a lot less steroid and a lot more antibiotic, in general terms. (ENT 08)

\section{Theme 3: decision-making for surgery Implementing guidelines}

ENT specialists keep up to date with current practices in CRS by reading rhinology journals and attending ENT conferences. All ENT specialists were familiar with the EPOS guidelines and described them as a good source of evidence for diagnosis and treatment decisions, although most general ENT surgeons did not refer to them on a daily basis.

So generally EPOS guidelines are probably the thing that I pay the most attention to. (ENT 09)

\section{Value of sinus surgery}

ENT specialists describe sinus surgery as an important treatment for patients with severe disease, and when medical treatment options have been explored.

Well, I think the patients at the more severe end of the spectrum, I think it offers them the best chance of a long-term improvement in symptoms. (ENT 05)

Similarly, GPs viewed sinus surgery as a treatment option which should be reserved for more severe patients who have tried all available medical treatments.

I think there is a role for surgery but I'm not someone who likes intervention. So, I would, initially, try all the things, the nasal therapies, basically, to see if that does the job first. Surgery is a last resort, for me. (GP 07)
However, some specialists describe uncertainties about the value of a surgical intervention for an inflammatory condition.

I'm not intellectually massively impressed by it, if I'm absolutely honest with you, the idea of doing an operation to help a mucosal inflammatory problem. (ENT 08)

ENT specialists identify the role of surgery in polyp disease and report prioritising these patients for surgical intervention.

The severe polyp patients, the ones who are completely bunged up, I wouldn't bother with any of the medical therapy. I just put them on my operating list for surgery. (ENT 05)

Both GPs and ENT specialists view surgery as a temporary rather than permanent solution for CRS. ENT specialists recount that most patients require ongoing medical treatment to manage symptoms after surgery, and GPs describe surgery as rarely a long-term solution for patients with CRS.

I mean I think it's rare that surgery is curative for those people, I would say, they don't just go and have one operation and never have any problems with their nose and sinuses for the rest of their life! These patients come back and back, even when they've had surgery. (GP 02)

\section{Joint decision-making}

ENT specialists reported that decision-making for surgery is made jointly with the patient after all treatment options, risks and potential benefits have been discussed.

It's very much up to the patients. Our patients are a very switched on bunch of people. They totally like to be involved in their decision. (ENT 09)

However, both GPs and ENT specialists recognise that some patients have a high expectation for surgery while others express a reluctance for surgery, especially repeat surgery.

Then there are some people who definitely want surgery from the outset, and don't want to have medical therapy, and they're difficult to manage because they won't accept a trial of medical therapy, because they've had antibiotics before and they've had those sprays and those drops, and they don't work. (ENT 01)

Some GPs describe advising patients against surgery due to the possible complications, side effects and potentially limited benefits.

I tend to counsel people fairly strongly against having sinus surgery because I just don't believe it's a longterm benefit in the vast majority of cases. (GP 04) 
Both GPs and ENT specialists recognise the potential risks of sinus surgery and describe the importance of balancing these against uncertain symptomatic relief for patients.

ENT surgery is painful, causes people to have a lot of time off work and may not yield high benefit and may make the problem worse. So I would have thought that that should come at a later stage, so not to cause harm. (GP 01)

\section{Theme 4: transition of care}

\section{Factors affecting referral}

GPs use their clinical judgement when deciding either to continue to manage a patient in primary care or to refer for specialist opinion and further treatment options. The impact of symptoms on a patient's quality of life and response to medical treatments were recognised by GPs as important factors for referral.

It really hinges, I think, on whether they're getting better with the simple treatment that we've put in place. If they're not getting better and it's affecting them and having effect on their functioning and their quality of life, then I would refer them. (GP 11)

GPs explained that patients with visible nasal polyps and those with structural abnormalities were prioritised for referral due to the potential need for surgical intervention.

I would refer someone if they had obstructing polyps that hadn't responded to medical polypectomy, assuming that they wanted to have a surgical polypectomy. (GP 04)

Some GPs report patient pressure for early referral, although many GPs report giving patients the choice about continued treatment in primary care or referral for a specialist opinion.

In some instances where they're adamant that they want to see a specialist, then we've had to write directly to the clinic and bypassing the referral to say that specifically this patient is wanting to see a specialist. (GP 08)

Many GPs were unaware of local referral guidelines for CRS and based their decision for referral on personal experience. However, others described a referral triage system or a local referral support service who screen the referral against local guidelines.

So, for the referral form, the guidelines are actually attached to that. So, at the point of referral, you can actually have a look at that and see if this patient fits or if there is more for you to do before it gets to ENT. (GP 07)

\section{Quality of referral}

ENT specialists report wide variation in the timing and quality of GP referrals to secondary care. Some specialists observed a delay in referral when symptoms are severe and suggest that earlier referral would be easier to treat and result in better outcomes for patients.

So I quite frequently see patients that have suffered symptoms for many months, if not years. It's a very frequent comment that I get from patients, saying I've been trying to get referred to a specialist for ages and ages and they just won't refer me and they just give me nose drops and sprays. (ENT 05)

Equally, other specialists report cases of premature referral, when patients have not received maximal available medical treatment in primary care.

I think I might criticise them for referring too presumptuously because I think we do get referrals from GPs who haven't actually even initiated medical therapy. (ENT 02)

\section{DISCUSSION \\ Synopses of key findings}

This study explored GPs and ENT specialist views of current practice for managing patients with CRS. GPs describe themselves as confident in recognising CRS with the exception of nasal polyps, however, surgeons report common missed diagnoses when patients are referred to ENT clinics and attribute this to the limited ENT training of GPs and lack of available diagnostic tests or equipment. Local prescribing restrictions can affect choice of INCS in primary care and poor adherence is perceived to be the causes of inadequate symptom control. Symptom severity, poor response to medical treatment and patient pressure drives referral, although there is lack of clarity about optimal timing. ENT clinic letters are a valuable information source for GPs and ENT training courses are useful but infrequent. Treatment decisions in secondary care are based on disease severity, polyp status, prior medical treatment and patient choice. Long-term antibiotic use is variable and specialists are uncertain about optimal dosing and which patients might benefit. Surgery is regarded as an important treatment option for patients with severe symptoms and especially in those with nasal polyps, although timing of surgery remains unclear, and the uncertainty about net long-term benefits of surgery makes balancing of benefits and risks more difficult.

\section{Comparison with current literature \\ GP management}

The findings of this study suggests that there is wide variation in the diagnosis and management of CRS in primary care in the UK, which is likely to be due to limited ENT training and insufficient national guidance. It has been long recognised that GPs receive limited ENT training at both undergraduate and postgraduate level ${ }^{16}$ and this 
is particularly important in light of the number of ENT conditions that are initially seen in general practice. Our findings agree with previous research that GPs would like further ENT training, ${ }^{17}$ and that regional courses and updates might be useful to improve knowledge, clinical skills and referral pathways to secondary care.

INCS are commonly prescribed for CRS; however, our research identified local prescribing restrictions and that both adherence and adequacy of correct nasal spray use by patients was perceived to be extremely variable. These findings support the results of a case-control study in the UK, which found that current INCS usage is $<15 \%$ in patients with CRS, and only $1 \%$ of patients regularly use saline nasal irrigation. ${ }^{18}$ Such usage may result in poor symptom control and untimely referral. Reasons for low usage are likely to be multifactorial. Previous qualitative work has identified that patients can be dissatisfied with topical treatments due to their perceived ineffectiveness ${ }^{19}$ and concern about potential side effects. ${ }^{20}$ Similarly, nasal irrigation was not widely advocated by GPs in this study due to uncertainties about effectiveness and a perceived burden for patients. However, there is now evidence from a recent trial which found nasal irrigation to be acceptable to patients with recurrent or chronic rhinosinusitis and provides symptomatic benefit in the primary care setting. ${ }^{21}{ }^{22}$ Currently in the UK, nasal irrigation kits cannot be prescribed by GPs as they are considered medical devices rather than medicines, so have to be purchased by patients and costs may deter uptake.

Some GPs described uncertainty about the optimal timing of referral, with wide variation in practice and a lack of clarity about referral criteria for both CRSsNP and CRSwNP. Correspondingly, ENT specialists reported variation in timing and precision of referral, expressing concerns that some patients experience unnecessarily delays, while others were referred too early and without trials of basic medical treatment. The EPOS guidelines ${ }^{3}$ recommend referral in patients where no improvement has been achieved after 4 weeks of treatment with INCS and nasal irrigation. However, knowledge and uptake of the European guideline is understandably variable in UK primary care. An audit of Clinical Commissioning Groups (CCGs) in England found that compliance with CRS commissioning was limited in $13 \%$ of CCGs. ${ }^{23}$ It may be that such guidance is not perceived in the same way that NICE guidance is viewed, affecting management and referral decisions, and fostering the variation of practice reported in this study. Similarly, in Dutch general practice, management of patients with CRS has been reported inconsistent and not always in accordance with local guidelines. $^{24}$

\section{ENT management}

Our study highlighted wide variation in diagnostic criteria and management decisions for CRS even among ENT specialists. Practice variation in usage of long-term antibiotics and timing of sinus surgery suggest that there is a lack of clarity around the evidence base for treatment of both CRSsNP and CRSwNP. There remains a paucity of evidence for sinus surgery, and more research is required to understand best timing, although emerging evidence shows that surgery undertaken closer to the time of diagnosis may improve long-term outcomes both in terms of symptoms and late-onset asthma. ${ }^{25}{ }^{26}$ There is also a perception among GPs and ENT specialists in this study that surgery was not of any long-term benefit and that patients will simply keep returning; this is of course borne out by the high revision rate seen in two UK national studies. ${ }^{27}{ }^{28} \mathrm{~A}$ key part of this issue is the postoperative care and compliance with topical treatment. The forthcoming trial in the MACRO programme ${ }^{14}$ will address the role of sinus surgery in a randomised controlled trial context which has not been done to date, despite many case series showing favourable outcomes, including the UK Sinonasal audit. ${ }^{29}$ There will also be a long-term plan to follow-up the patients beyond the trial so that the benefits of sinus surgery beyond the short-term can be addressed.

\section{Implications for practice}

In view of the currently fragmented and diverse situation, there is a need to clarify care pathways for CRSwNP and CRSsNP across primary and secondary care. Development of an evidence-based integrated care pathway, informed by patient, generalist and specialist perspectives, may help to improve management of patients with CRS. Communication between ENT and general practice needs enhancing, and clarification of diagnostic, treatment and referral algorithms has the potential to improve early management and precision of referral. Improving the information to both patients and clinicians as to the appropriate use of both medical and surgical interventions to best effect, including addressing rationale for treatment options, safety, technique, compliance and dispelling misperceptions around the various treatment options, has the potential to improve outcomes and reduce variation and costs.

\section{Strengths and limitations}

A key strength of this study was the inclusion of both generalist and specialist views from representative samples of frontline clinicians, to provide a better understanding of the CRS patient pathway from multiple perspectives, which have not been captured in previous studies. However, it is possible that our sample of clinicians were particularly interested in CRS or research of this nature and thus their views may not have represented those of the non-respondents.

A good level of thematic data saturation was achieved with our sample of participants, but we acknowledge that the views of other stakeholders such as nurse practitioners in primary care and ENT specialty doctors may have provided an additional viewpoint.

Additionally, the views and experiences of patients with CRS are important in understanding the patient journey and these are presented elsewhere. 
This study used rigorous methods to ensure credibility and trustworthiness of the findings, including multiple coders, constant comparison techniques and maintained a transparent audit trail.

\section{CONCLUSION}

In summary, this qualitative study found that clinicians are uncertain about best management of patients with CRS in both primary and secondary care, and there is wide variation in practice. Improved communication between ENT and general practice together with an evidence-based integrated care pathway for CRSsNP and CRSwNP is needed to improve CRS patient management and timely referral.

\section{Author affiliations}

${ }^{1}$ Primary Care and Population Sciences, Faculty of Medicine, University of

Southampton, Southampton, UK

'Guy's and St. Thomas' NHS Foundation Trust, London, UK

${ }^{3}$ evidENT, University College London, London, UK

${ }^{4}$ Fifth Sense: the charity of people with smell and taste disorders, Chinnor, Oxfordshire, UK

${ }^{5}$ Norwich Medical School, University of East Anglia, Norwich, UK

${ }^{6}$ James Paget University Hospitals NHS Foundation Trust, Great Yarmouth, UK

Acknowledgements The authors would like to thank the healthcare professionals who contributed and made this study possible.

Contributors The protocol was developed by CE, MT, CMP, CH, AS, PL and HB. JV led the data collection and analysis, with academic contributions from CE, MT, CMP and $\mathrm{CH}$. JV drafted the manuscript and coordinated the revisions from all authors. All authors read and approved the final manuscripts. A patient contributor (JB) from the MACRO programme management group also provided input into the design of the study, and reviewed and commented on drafts of this manuscript.

Funding This paper presents independent research funded by the National Institute for Health Research (NIHR) under its Programme Grants for Applied Research (PGfAR) Programme (Grant Reference Number RP-PG-0614-20011).

Disclaimer The views expressed are those of the author(s) and not necessarily those of the NHS, the NIHR or the Department of Health.

Competing interests None declared.

Patient consent Not required.

Ethics approval The study was given ethical approval by the Health and Social Care Research Ethics Committee A (HSC REC A) on 22 September 2017 (16/ NI/0197).

Provenance and peer review Not commissioned; externally peer reviewed.

Data sharing statement There is no additional data available for sharing.

Open access This is an open access article distributed in accordance with the Creative Commons Attribution Non Commercial (CC BY-NC 4.0) license, which permits others to distribute, remix, adapt, build upon this work non-commercially, and license their derivative works on different terms, provided the original work is properly cited, appropriate credit is given, any changes made indicated, and the use is non-commercial. See: http://creativecommons.org/licenses/by-nc/4.0/.

\section{REFERENCES}

1. Gliklich RE, Metson R. The health impact of chronic sinusitis in patients seeking otolaryngologic care. Otolaryngol Head Neck Surg 1995;113:104-9.

2. Wensing M, Vingerhoets E, Grol R. Functional status, health problems, age and comorbidity in primary care patients. Qual Life Res 2001;10:141-8.

3. Fokkens WJ, Lund VJ, Mullol J, et al. EPOS 2012: European position paper on rhinosinusitis and nasal polyps 2012. A summary for otorhinolaryngologists. Rhinology 2012;50:1-12.
4. Hastan D, Fokkens WJ, Bachert C, et al. Chronic rhinosinusitis in Europe--an underestimated disease. A GA2LEN study. Allergy 2011;66:1216-23.

5. Hopkins C, Holy C, Philpott C. Care pathway of patients with chronic rhinosinusitis in the UK. Liverpool: British Academic Conference in Otorhinolaryngology, 2015.

6. Department of Health. Hospital Episode statistics, 2013.

7. Desrosiers M, Evans GA, Keith PK, et al. Canadian clinical practice guidelines for acute and chronic rhinosinusitis. Allergy Asthma Clin Immunol 2011;7:2

8. Royal College of Surgeons. Commissioning Guide. 2013 https:// www.rcseng.ac.uk/standards-and-research/commissioning/ commissioning-guides/topics/.

9. Chong LY, Head K, Hopkins C, et al. Different types of intranasal steroids for chronic rhinosinusitis. Cochrane Database Syst Rev 2016;4:Cd011993.

10. Chong LY, Head K, Hopkins $\mathrm{C}$, et al. Intranasal steroids versus placebo or no intervention for chronic rhinosinusitis. Cochrane Database Syst Rev 2016;4:Cd011996.

11. Chong LY, Head K, Hopkins C, et al. Saline irrigation for chronic rhinosinusitis. Cochrane Database Syst Rev 2016;4:Cd011995.

12. Gulliford MC, Dregan A, Moore MV, et al. Continued high rates of antibiotic prescribing to adults with respiratory tract infection: survey of 568 UK general practices. BMJ Open 2014;4:e006245.

13. Lasso A, Masoudian P, Quinn JG, et al. Long-term low-dose macrolides for chronic rhinosinusitis in adults - a systematic review of the literature. Clin Otolaryngol 2017;42:637-50.

14. The MACRO programme. https://www.themacroprogramme.org.uk/ (accessed Jan 2018).

15. Braun V, Clarke V. Using thematic analysis in psychology. Qualitative Research in Psychology 2006;3:77-101.

16. Clamp PJ, Gunasekaran S, Pothier DD, et al. ENT in general practice: training, experience and referral rates. $J$ Laryngol Otol 2007;121:580-3.

17. Easto RH, Reddy V. A survey of ENT experience in South West Peninsula general practitioner trainees: how can post-graduate ENT training be improved? J Laryngol Otol 2016;130:893-6.

18. Philpott C, Erskine S, Smith R, et al. Current use of baseline medical treatment in chronic rhinosinusitis: Data from the National Chronic Rhinosinusitis Epidemiology Study (CRES). Clin Otolaryngol 2018;43:509-24.

19. Erskine SE, Verkerk MM, Notley C, et al. Chronic rhinosinusitis: patient experiences of primary and secondary care - a qualitative study. Clin Otolaryngol 2016;41:8-14.

20. Hellings PW, Dobbels F, Denhaerynck K, et al. Explorative study on patient's perceived knowledge level, expectations, preferences and fear of side effects for treatment for allergic rhinitis. Clin Transl Allergy 2012;2:9.

21. Little P, Stuart B, Mullee M, et al. Effectiveness of steam inhalation and nasal irrigation for chronic or recurrent sinus symptoms in primary care: a pragmatic randomized controlled trial. CMAJ 2016;188:940-9.

22. Leydon GM, McDermott L, Thomas T, et al. 'Well, it literally stops me from having a life when it's really bad': a nested qualitative interview study of patient views on the use of self-management treatments for the management of recurrent sinusitis (SNIFS trial). BMJ Open 2017; 7:e017130.

23. Soni-Jaiswal A, Philpott C, Hopkins C. The impact of commissioning for rhinosinusitis in England. Clin Otolaryngol 2015;40:639-45.

24. Hoffmans R, Schermer T, van Weel C, et al. Management of rhinosinusitis in Dutch general practice. Prim Care Respir $J$ 2011;20:64-70.

25. Hopkins C, Rimmer J, Lund VJ. Does time to endoscopic sinus surgery impact outcomes in Chronic Rhinosinusitis? Prospective findings from the National Comparative Audit of Surgery for Nasal Polyposis and Chronic Rhinosinusitis. Rhinology 2015;53:10-17.

26. Benninger MS, Sindwani R, Holy CE, et al. Impact of medically recalcitrant chronic rhinosinusitis on incidence of asthma. Int Forum Allergy Rhinol 2016;6:124-9.

27. Hopkins C, Slack R, Lund V, et al. Long-term outcomes from the English national comparative audit of surgery for nasal polyposis and chronic rhinosinusitis. Laryngoscope 2009;119:2459-65.

28. Philpott $\mathrm{C}$, Hopkins $\mathrm{C}$, Erskine $\mathrm{S}$, et al. The burden of revision sinonasal surgery in the UK-data from the Chronic Rhinosinusitis Epidemiology Study (CRES): a cross-sectional study. BMJ Open 2015;5:e006680.

29. Hopkins C, Browne JP, Slack R, et al. The national comparative audit of surgery for nasal polyposis and chronic rhinosinusitis. Clin Otolaryngol 2006;31:390-8 